\title{
Leaders
}

\section{Is the proficiency test in cervical cytology proficient?}

\author{
C J R Stewart
}

It is accepted that internal quality control and external quality assurance are necessary elements in ensuring high standards in the reporting of cervical smears. A major component of external quality assurance in the United Kingdom in recent years has been the proficiency test in cervical cytology. Participation in regional proficiency test schemes was recommended for all staff in recently published documents concerned primarily with internal quality control in cytology laboratories. ${ }^{12}$ Furthermore, the working party assessing quality assurance guidelines for the cervical screening programme used participation and acceptable performance in the UK proficiency test scheme as a measure of its objective "to ensure that all staff screening or reporting smears are competent". ${ }^{3}$ The combined weight of these authoritative documents suggests that the rationale for proficiency test must be beyond question. However, at a time when proficiency test is under review, both in the United Kingdom and the United States, it may be worth considering the evidence that proficiency test actually improves laboratory performance.

The regional proficiency test schemes in the UK have evolved from a protocol published in 1988 under the auspices of the Department of Health Advisory Committee on Assessment of Laboratory Standards. ${ }^{4}$ This document referred to the successful proficiency test programmes operational in North America, and commented that the protocol UK test was based on the New York Proficiency Test Scheme (NYPTS). It should be emphasised, however, that there are significant differences between the NYPTS and the UK proficiency testing programmes. The test slide set in the NYPTS comprises only one element of the proficiency testing scheme, the others, which are arguably more important, being laboratory inspection and an educational programme, more analogous to clinical pathology accreditation and circulating slide sets, respectively, in the $\mathrm{UK}^{.}{ }^{5-7}$ In addition, the examination itself differs from those in the UK by including nongynaecological material (comprising $50 \%$ of the test set since 1982). ${ }^{7}$ Furthermore, the testing mechanism does not necessarily involve all reporting staff. Only one pathologist need review the slides in each laboratory, these being pre-assessed and marked by the cytotechnology staff. The rationale of this approach is to mimic daily practice as closely as possible and the laboratory performance is judged on the final diagnosis offered by the pathologist. It is of course debatable whether the UK programmes offer an improvement on the NYPTS but comparison of data is of doubtful validity.

\section{North American schemes}

Notwithstanding these concerns, the North American proficiency test programmes have all shown improved laboratory pass rates with time. ${ }^{78}$ However, none has shown that these results correlate in any way with actual laboratory performance in routine cases. As Austin points out, "there is virtually no scientific evidence that regulatory proficiency testing decreases interpretative false negative rates". Indeed there is considerable difficulty in assessing the effect of proficiency test given that it is only one component of laboratory quality assurance. Austin studied cervical cancer death rates in the USA and showed that New York had actually fallen behind other states in preventing deaths since the inception of NYPTS. ${ }^{9}$ Similarly the death rate from cervical cancer in Ontario, which began a proficiency test scheme in 1978, had not declined more quickly than those in the other Canadian provinces (which have not introduced such schemes). ${ }^{9}$ However, use of the cervical cancer death rate as an indicator of the value of proficiency test has been criticised as it is significantly influenced by numerous other factors. ${ }^{10}$ The false negative error rate in cervical smear reporting appears to be a more relevant indicator of laboratory performance. A Q-probes analysis by the College of American Pathologists (CAP) examined the false negative error rate in 353 laboratories based on a review of previous "negative" smears in patients presenting with high grade squamous intraepithelial lesions. No difference in the laboratory error rate was found according to participation in a proficiency test scheme. ${ }^{11}$
Castle Street, Glasgow

C J R Stewart

Accepted for publication 8 April 1997 


\section{UK studies}

There are few studies examining critically the effect of proficiency test in the UK. Gifford and Coleman described a scheme involving 17 laboratories in the North West Thames region. ${ }^{12}$ One hundred and forty six cytotechnologists and pathologists participated in the scheme that comprised four rounds of testing. Although $95 \%$ of participants achieved a satisfactory score, three, including two consultants and one cytotechnologist, did not reach an acceptable level of performance. One individual was deemed unsuitable to report cervical cytology, another was advised to undergo retraining and retesting, and the third volunteered for early retirement. After this action was taken it was noted that the subsequent laboratory performance was "undoubtedly improved". As with the North American studies, this improvement appears to have been related solely to performance in proficiency tests. It may be that these cytologists' performances in routine practice were also substandard but the study failed to provide this information. This is critical to determining the validity of proficiency testing. For example, if it had been previously recognised that those concerned were performing at a suboptimal level based on internal quality control data, then did the proficiency test actually contribute further information? One might assume that any necessary action would have been taken prior to the proficiency test results. Alternatively, if the individuals had been performing routinely at a satisfactory level, should the results of the proficiency test be considered more important and relevant than the performance data gathered over extended periods in the real working environment? The authors of this paper also commented that the proficiency test scheme had confirmed the very high standard of screening in the North West Thames region. ${ }^{12}$ This may have been the case but no data were presented to support this conclusion. The mistaken assumption that satisfactory proficiency test performance is evidence of high clinical standard has previously been made with regard to US schemes; however, proficiency testing has never been proven to improve diagnostic accuracy. ${ }^{13}$ As Bachner notes, "no evidence has ever been presented that mandated cytology proficiency test can lead to or has led to an improvement in routine clinical practice that is linked to improvement in patient care". ${ }^{14}$

A second UK study by Slater examined external and internal quality assurance in the Trent region. ${ }^{15}$ It was noted that one cytoscreener, who had always passed the proficiency test, appeared to be making relatively frequent reporting errors. This led to a rescreening exercise which detected a $15 \%$ false negative rate by the individual, which was higher than that of other cytoscreeners and was considered marginal performance. Therefore, the proficiency test failed to identify suboptimal performance in this cytoscreener and, as Slater conceded, "indeed conveyed a false impression that no problem existed". The Cytopathology Education Consortium (CEC), comprising the American Society of Clinical Pathologists, the American Society of Cytopathology, the American Society of Cytotechnology, and the CAP, have likewise recently concluded that "there is no assurance that marginal practitioners (of gynecological cytology) are reliably identified by means of a 10 -slide regulatory proficiency test" ${ }^{16}$ Slater also commented that in his view "proficiency testing has an important role in the identification of overtly poor performance". ${ }^{15}$ Is this the purpose of the proficiency test? Might it not be expected that an effective internal quality control programme would detect any individual making such overt errors given that even marginally suboptimal performance was identified in the case he presented?

\section{Disadvantages of proficiency testing}

Any assessment of a proficiency test must also consider its disadvantages, including cost, lack of relevance, and effects on professional morale. The costs of proficiency testing are difficult to assess but are not simply those attributable to administrative overheads and the disruption to, and loss of, laboratory working time, important as these are at a time of increasing financial constraint and work load pressures. The stress of proficiency testing on cytology staff should also be considered, as should the effect on overall laboratory morale. Individuals sitting the proficiency test are well aware that failure could ultimately lead to loss of employment, particularly if service purchasers make satisfactory performance in the test a condition of contracts. The apprehension associated with the proficiency test was conceded in the original UK protocol ${ }^{4}$ although it was felt that there would also be reassurance, presumably in the event of a pass. It might prove interesting to examine the laboratory error rates in cervical smear reporting as assessed by internal quality control around the time of proficiency testing compared with average values.

Perhaps the greatest weakness of proficiency testing is the assumption that examination of a test slide set compares in any way with normal screening practice. The test environment is artificial and the slides inevitably include a much higher proportion of abnormal smears than could be expected in normal screening practice. For example, in the Ontario proficiency test, smears showing dysplasia or malignancy accounted for $26-46 \%$ of slides, ${ }^{8}$ and personal experience suggests at least a similar proportion of abnormals in the Scottish scheme. Thus, and as admitted by Curry et al, the test does not reflect daily practice conditions. ${ }^{8}$ There are often significant differences in staining between laboratories which may cause difficulties in interpretation. In addition candidates are unable to dot and review abnormal cells before making a diagnosis. Finally, and most obviously, no discussion of slides is permitted between staff. The usual argument against these criticisms is that the test slides are selected to be unequivocal diagnostic examples. Surely, however, this is a self defeating argument if there is any pretence that the test is supposed to simulate normal 
practice. Even proponents of proficiency testing accept its limited value in this respect. Melamed, with reference to the NYPTS, commented that "the test situation is obviously quite different from the normal laboratory setting and I suspect that it does not really reflect normal laboratory function". ${ }^{17}$ Plott and colleagues emphasised that "screening results from this simulated test should not be extrapolated to routine work performance". ${ }^{18}$ Davey summarised the view of the 1993 proficiency test symposium in Atlanta, Georgia, USA that concluded, "there was solid agreement that proficiency testing measures only limited aspects of performance and may not correlate with day to day screening or actual patient outcome". ${ }^{19}$ More recently, the CEC argued that proficiency testing should be directed to laboratories as a whole rather than to individuals. According to the CEC, regulatory testing of individuals "does not simulate normal practice, will be unnecessarily disruptive, and has not shown to decrease clinically significant errors or improve screening outcomes". ${ }^{16}$

Lowering of professional morale is an inevitable consequence of the requirement that individuals involved in cervical screening must pass the proficiency test. The test effectively supplants previous qualifications, performance record, and professional standing that are often based on many years' continual peer review and contact with colleagues in the laboratory, with hospital clinicians, and with general practitioners. Bachner ${ }^{14}$ commented that the title proficiency test is in fact a misnomer and that recredentialing would be a more appropriate term. Austin" likewise pointed out that "proficiency testing of individual cytologists is a defacto recertification program which usurps the authority" of the licensing boards in the US. The CEC has also concluded that regulatory cytopathology testing would be a recertification programme that would inappropriately subsume the authority of the various US professional bodies. ${ }^{16}$ All those involved in the examination of cervical smears in the UK have similarly undergone appropriate training, examination and, more recently, involvement in continuing medical education and continuing professional development. Is it appropriate that these qualifications and evidence of ongoing education should be of lesser importance than the proficiency test?

\section{Legal ramifications}

The current status of proficiency testing in the UK has another potential complication, namely the legal ramifications should any individual choose to pursue the validity of the test in the court of law. Valente ${ }^{20}$ pointed out that "if proficiency testing is to serve a regulatory function it may potentially deprive someone of the ability to earn a livelihood. Therefore it must be fair and legally defensible". It is surely open to question whether the proficiency test would survive legal analysis based on current evidence. Perhaps pathology staff in the UK should take note of the problems encountered in the US following the decision to implement a national cytology proficiency testing pro- gramme in the Clinical Laboratory Improvement Amendments (CLIA) of $1988 . .^{21}$ As of 1995 only the State of Maryland cytology proficiency testing scheme met the CLIA cytology requirements, and only one other state application had been received, this being subsequently withdrawn. ${ }^{22}$ Ironically, the US Department of Health has actually been forced to increase the stringency of proficiency testing following a successful lawsuit by the Consumer Federation of America. The court order stipulated that proficiency test slides be examined at a working rate of 12.5 slides per hour rather than five slides per hour to conform with normal working conditions. ${ }^{22}$ It is pertinent that the maximum workload limit was initially deemed unsuitable "since there are salient differences between the routine examination of patient material and cytology proficiency testing". The potential legal consequences of proficiency testing therefore could arise either from its unfairness to laboratory staff or from its perceived inadequacy by the public.

\section{Alternatives}

If external quality assessment is essential then what are the alternatives to the proficiency test? The CEC has proposed that mailed regulatory challenges aimed at the laboratory as a whole rather than individuals be combined with internal education based exercises. ${ }^{16}$ In their view the laboratory director would be responsible for assuring the competency of individuals and for taking remedial action if required. It seems possible that an external assessor/ facilitator could oversee such assessments as well as review internal quality control data to ensure independent inspection. The development of computer based technology is also likely to be vital in both teaching and proficiency evaluation. ${ }^{2123}$ Slide circulation schemes have previously been used in external quality assessment and, while these also suffer from disadvantages, their educational value is higher than proficiency testing. ${ }^{24}$ Indeed, the emphasis on education rather than testing to maintain and improve standards in cervical cytology are agreed by most authors. The overall reporting rates offer an additional useful guide to the assessment of laboratory performance. ${ }^{25}$

It remains to be seen whether the UK proficiency test will persist in its current form or whether alternative methods of external quality assessment will be considered. Given that the evidence in favour of proficiency testing is currently lacking, it is hoped, at a minimum, that any continuity of the UK proficiency testing programme will include an analysis of its purported benefit, independent of internal quality control. Otherwise the credibility of the proficiency test will continue to be questioned.

1 Herbert A, Johnson J, Patnick J, Burtenshaw A, Codling BW, Day NE, et al. Achievable standards, benchmarks for reporting, criteria for evaluating cervical cytopathology. Cytopathology 1995;6(Suppl 2):7-32.

2 Hennigan M, Keel A, Campbell GM, Forbes JF, Gray W, Imrie J, et al. Report of working party on internal quality control for cervical cytopathology laboratories. Edinburgh: The Scottish Office, 1995. 
3 Pritchard J, Patnick J, Austoker J, Blaney J, Chishty V, Duncan I, et al. Quality assurance guidelines for the cervical screening programme. NHSCSP No. 3, 1996.

4 Hudson E, Brooke D, Brown C, Gauntlett J, Lader S, Light $\mathrm{AM}$, et al. Protocol for a proficiency testing scheme in gynaecological cytopathology. Department of Health Advisory Committee on Assessment of Laboratory Standards, 1988.

5 Collins DN, Kaufmann W, Albrecht R. New York State computerised proficiency testing program in exfoliative computerised proficiency testing program in exfol

6 Collins DN, Kaufmann W, Clinton W. Quality evaluation of cytology laboratories in New York State; expanded program, 1971-73. Acta Cytol 1974;18:404-13.

7 Collins DN, Patacsil DP. Proficiency testing in cytology in New York. Analysis of a 14-year state program. Acta Cytol 1986;30:633-42.

8 Curry H, Thompson DW, Dietrich M, Lipa M, Massarella GR, Taves IR, et al. Proficiency testing in cytology laboratories in Ontario, Canada: a decade of experience. Acto Cytol 1987;31:215-19.

9 Austin RM. Can regulatory proficiency testing by the cytobureaucracy decrease both false negatives and cervical canbureaucracy decrease both false negatives and

10 Nagy GK, Collins DN, Newton LE. Proficiency testing in cytology-does it have any value? Diagn Cytopathol 1995;12:381-2.

11 Jones BA, Heard NV. Pap smear rescreening. Data analysis and critique. Chicago: College of American Pathologists, 1993.

12 Gifford C, Coleman DV. Quality assurance in cervical cancer screening: results of a proficiency testing scheme for cytology laboratories in the North West Thames region. Cytopathology 1994;5:197-206.

13 Valente PT. Cytology proficiency testing. Mandated method in search of validation. In: ASCP reviews in pathology. in search of validation. In: ASCP reviews in pathology:
14 Bachner P. Can cytology proficiency testing programs discriminate between competent and incompetent practitioners? Qual Rev Bull 1991;17:150-1.

15 Slater DN. Cervical cytology external and internal quality assurance: a comparative appraisal. f Clin Pathol 1995;48: 95-7.

16 CEC proposal on proficiency testing. ASC Bull 1996;23:42-

17 Melamed MR. Presidential address. Twentieth annual scientific meeting, American Society of Cytology. Acta Cytol 1973;17:285-8.

18 Plott AE, Martin FJ, Cheek SW, Yobs AR, Wood RJ. Measuring screening skills in gynecologic cytology. Results of voluntary self-assessment. Acta Cytol 1987;31:911-23.

19 Davey DD. On the American scene: cytology proficiency testing symposium overview. Diagn Cytopathol 1994;10: 198-9.

20 Valente PT. Government mandated cytology proficiency testing: time for reality testing. Diagn Cytopathol 1994;10: 105-6.

21 Voelker R. Cytology proficiency testing has stumped experts. $\mathcal{F} A M A$ 1993;270:2779-83.

22 CLIA program; cytology proficiency testing. Washington DC: Federal Register 1995;61:509.

23 Rashbass J, Vawer A. A networked computer program for managing a national external quality assurance scheme in cytopathology. Cytopathology 1996;7:377-85.

24 Boon AP, Williamson P, Brown JM, Thomas GDH. The Yorkshire slide exchange external quality assessment (EQA) scheme. Cytopathology 1996;7:90-8.

25 Butland D, Herbert A. Comparison of cervical cytology reporting rates: a useful adjunct to external quality assurance. Cytopathology 1996;7:391-9.

\section{Molecular Pathology-June 1997 contents}

\section{Reviews}

Functional and clinical aspects of the myelomonocyte protein calprotectin

B fohne, M K Fagerhol, T Lyberg, H Prydz, P Brandtzag, C F Naess-Andresen, I Dale

Isoenzymes of protein kinase $\mathrm{C}$ : differential involvement in apoptosis and pathogenesis

E M Deacon, $\mathcal{F}$ Pongracz, G Griffiths, $\mathcal{F} M$ Lord

Apoptosis in bone physiology and disease

$D E$ Hughes, $B$ F Boyce

\section{Papers}

Expression of the Wilms' tumour gene WT1 in the developing human and in paediatric renal tumours: an immunohistochemical study

A K Charles, $S$ Mall, $\mathcal{F}$ Watson, P $\mathcal{f}$ Berry

Human papillomavirus and schistosomiasis associated bladder cancer

$K$ Cooper, $Z$ Haffajee, $L$ Taylor

Nucleoli and AgNORs in Hodgkin's disease

N N Mamaev, $N$ V Medvedeva, V F Shust, A B Markochev, N D Pasternak

"Aggrecanase" activity is implicated in tumour necrosis factor $\alpha$ mediated cartilage aggrecan breakdown but is not detected by an in vitro assay

$D \mathcal{F}$ Buttle, $A$ Fowles, $M Z$ Ilic, $C \mathcal{F}$ Handley

In situ end labelling: effect of proteolytic enzyme pretreatment and hydrochloric acid $N \mathcal{F}$ Carr, I C Talbot

\section{Short report}

Modification of IgH PCR clonal analysis by the addition of sucrose and cresol red directly to PCR reaction mixes

$E$ Hodges, $S M$ Boddy, $S$ Thomas, $\mathcal{F} L$ Smith

\section{Miscellanea}

Book reviews $\bullet$ Correction $\bullet$ Notices 